
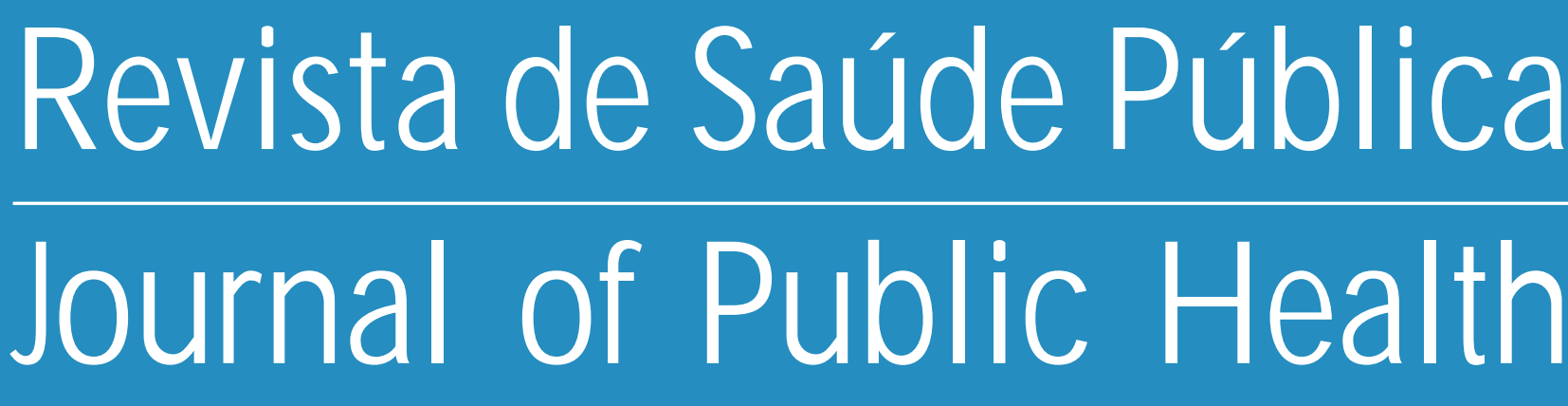

Avaliação da qualidade dos dados populacionais e cobertura dos registros de óbitos para as regiões brasileiras*

Evaluation of population data quality and coverage of registration of deaths for the Brazilian regions

Neir Antunes Paes e Marconi Edson Esmeraldo Albuquerque

Departamento de Estatística do Centro de Ciências Exatas e da Natureza da Universidade Federal da Paraíba. João Pessoa, PB - Brasil 


\section{Avaliação da qualidade dos dados populacionais e cobertura dos registros de óbitos para as regiões brasileiras*}

\section{Evaluation of population data quality and coverage of registration of deaths for the Brazilian regions}

\section{Neir Antunes Paes e Marconi Edson Esmeraldo Albuquerque \\ Departamento de Estatística do Centro de Ciências Exatas e da Natureza da Universidade Federal da Paraíba. João Pessoa, PB - Brasil}

\section{Descritores}

Censos. Estatísticas vitais.

Registros de mortalidade.

Controle de qualidade.

\section{Keywords}

Censuses. Vital statistics.

Mortalitiy registries. Quality control.

\section{Resumo}

Objetivo

Avaliar a qualidade dos dados populacionais e a cobertura dos registros de óbitos para as Unidades da Federação Brasileira (UF), por sexo, no ano de 1990.

\section{Métodos}

Os dados populacionais extraídos dos Censos Demográficos foram avaliados através de métodos demográficos clássicos. Os registros de óbitos, extraídos da Fundação Instituto Brasileiro de Geografia e Estatística e do Ministério da Saúde, foram avaliados através de três técnicas de mensuração do sub-registro de óbitos.

\section{Resultados}

A precisão das declarações das idades das UF, em sua maioria, passou do status de "pouco preciso" ou "moderado" para "preciso" na década de 80. A cobertura dos óbitos, em 1990, foi classificada como "boa" ou "satisfatória" para todos os Estados do Sul, Sudeste, Centro-Oeste e para os do Nordeste, abaixo do Rio Grande do Norte. O restante dos estados foram classificados como "regular" ou "insatisfatório".

\section{Conclusões}

Houve uma melhora importante na qualidade das declarações da população recenseada e aumento da cobertura de óbitos e que, para muitas UF, é possível gerar confiáveis indicadores de mortalidade.

\section{Abstract}

\section{Objective}

The evaluation of the quality of population data and coverage of death statistics for all Federal Brazilian Units by sex in 1990.

\section{Methods}

The population data came from censuses and the recorded death data from "Fundação Instituto Brasileiro de Geografia e Estatística” and the Health Ministry.

* Realizado com o apoio financeiro do Conselho Nacional de Desenvolvimento Científico e

Correspondência para/Correspondence to: Neir Antunes Paes Tecnológico/CNPq (Processo n ${ }^{\circ}$ 523355/94-5)

Recebido em 21.1.1998.

Reapresentado em 9.5.1998.

Aprovado em 3.8.1998. 
The population data were evaluated by applying classical demographic methods. Three techniques were chosen to evaluate the extent of death registration coverage.

Results

The degree of precision of the age statement for the majority of the Brazilian regions improved the status from "low precision" or "moderate" to "precise" during the 80's. The coverage of deaths in 1990 was classified as "good" or "satisfactory" for all Federal Units in the South, Southeast and Centre-West and for the Northeastern States below Rio Grande do Norte. All the remaining states were classified as "regular" or "unsatisfactory".

\section{Conclusions}

There was a significant improvement in the quality of the census population data and an increase in the coverage of death. It is possible to obtain get reliable mortality indicators for many Brazilian States.

\section{INTRO DUÇÃO}

As declarações censitárias das idades de uma população, classificadas por sexo, estão sujeitas a erros que, dependendo de sua magnitude podem comprometer a veracidade dos indicadores que delas dependem. Há dois tipos principais de erros: os que se referem à contagem, seja por omissão ou por contagem de um indivíduo inúmeras vezes, conseqüentes da má cobertura do censo, e os obtidos por falhas nas declarações, representados pela omissão ou declaração errônea. Sendo estas variáveis de uso freqüente na geração de indicadores demográficos e socioeconômicos, torna-se imprescíndivel uma avaliação da qualidade dessas declarações, da magnitude e do dimensionamento dos eventuais erros existentes.

Os registros dos fatos vitais no Brasil, por sua vez, são fontes de preocupação, principalmente por parte dos demógrafos e epidemiologistas, devido à qualidade duvidosa dos dados, os quais se constituem em entraves na obtenção de estatísticas confiáveis de mortalidade, dificultando, assim, as ações da saúde pública do País.

São poucos os trabalhos na literatura que se detiveram a estimar o sub-registro das declarações de óbitos. Geralmente, quando feitos, eles se detiveram a enfocar regiões isoladas ou agregações de unidades geográficas com metodologias diferentes. A disponibilidade das várias técnicas surgidas nas duas últimas décadas exige que se lance mão delas e se averigüe, conjuntamente para todas as unidades federativas do Brasil, quais são as mais adequadas no tocante às exigências dos supostos, e que proveito se pode tirar delas. Não se pretende fazer uma discussão teórica dos procedimentos, mas sim um estudo aplicativo de alguns deles para avaliar tanto a fidedignidade dos dados da população censitária em 1980 e 1991 como o nível de cobertura dos registros de óbitos para todos os estados da federação e o Brasil como um todo, em 1990. Objetiva-se também averigüar o quanto são comparáveis os dados de óbitos divulgados pela Fundação Instituto Brasileiro de Geografia e Estatística (FIBGE) e pelo Ministério da Saúde (MS). Estas informações de população e de óbitos, em conjunto, possibilitam a construção de taxas de mortalidade cujo estudo é feito, apenas, para o Brasil.

\section{MÉTODOS}

\section{Fontes de Dados}

As duas principais fontes oficiais de disseminação contínua das estatísticas de óbitos no Brasil são a FIBGE e o MS. Os dados da primeira são publicados na "Estatísticas do Registro Civil" e da segunda na "Estatísticas de Mortalidade"". Estas estatísticas, por diversas razões, não coincidem e, conseqüentemente, ambas as fontes exibem coberturas de óbitos diferenciadas. Os dados de população, por sua vez, foram extraídos dos censos demográficos ${ }^{3,4}$.

\section{Avaliação dos Dados Populacionais Censitários}

Para avaliar a qualidade das declarações das idades das populações classificadas por idade e sexo, para o Brasil e estados nos anos censitários de 1980 e 1991, são usados três métodos que indicam o nível de qualidade desses dados. A seguir está apresentada uma breve descrição deles. Uma boa revisão desses métodos pode ser encontrada no Manual II das Nações Unidas ( Naciones Unidas, 1995) ou ainda em compêndios condensados. ${ }^{12}$ 
O Índice das Nações Unidas é obtido através de uma combinação linear de dois outros: o desvio médio da razão de sexo e o desvio médio da razão de idade. O primeiro deles é definido através da média das diferenças sucessivas entre os índices de masculinidade (a razão entre a quantidade de homens e mulheres), calculados para cada grupo de idade e indica quanta variabilidade existe entre os grupos. E o segundo deles é definido pela média dos desvios, com relação a 100, das razões de idade, calculadas para cada um dos grupos etários, e detecta erros devido a transferência de um grupo a outro. Este índice é calculado para ambos os sexos

$\mathrm{O}$ Índice de Whipple mede a preferência digital e identifica a atração pelos dígitos 0 e 5, que são preferencialmente os dígitos mais declarados quando se arredondam dados. Ele é calculado para ambos os sexos e usa o princípio da retangularidade ou da linearidade para intervalos qüinqüenais ou decenais.

O Índice de Myers mede a atração ou repulsão por cada um dos 10 dígitos, de 0 a 9 . O princípio empregado consiste em determinar a proporção da população cuja idade termina em um dígito particular com relação ao total populacional que é multiplicada por um fator 0,1 (que representa a proporção esperada da população cuja idade termina em um dígito particular).

\section{Avaliação da Cobertura dos Registros de Óbitos}

Pelo menos dez métodos na literatura mundial foram propostos nas duas últimas décadas, os quais permitem estimar o grau da cobertura dos óbitos. A aplicação deles sugere que mesmo dados deficientes podem se constituir em referências úteis para estimar os níveis e tendências da mortalidade. Contudo, esses métodos diferem quanto aos supostos, exigências e grau de precisão. Não há nenhuma maneira mecânica de usá-los de modo a captar a sensibilidade e tipos de erros presentes nos dados. Uma ampla discussão sobre o uso deles para o caso brasileiro pode ser encontrado, por exemplo, em Paes $^{9}$, e para uma população genérica, em Preston e col. ${ }^{10}$ Entre eles, três métodos foram selecionados, no presente trabalho, para avaliar o nível do sub-registro de óbitos. Para o Brasil e estados, para o ano de 1990, a escolha deles foi baseada na flexibilidade de uso, suposições e exigências quanto aos dados básicos. Todos eles compartilham de uma característica comum: estimam a cobertura dos óbitos a partir de uma certa idade, no mínimo 5 anos de idade. Os princípios e metodologia de cada método pode facilmente ser encontrada nas compilações feitas no Manual X, elaborado pelas Nações Unidas ${ }^{13}$ que contém uma boa revisão deles; apenas breves considerações são feitas aqui.

O Método da Equação de Balanço do Crescimento foi desenvolvido por Brass ${ }^{1}$, o qual mostrou que em uma população estável, a equação do balanço do crescimento pode ser expressa como uma relação linear entre a taxa de mortalidade e a taxa de natalidade para os diferentes grupos etários, cujo coeficiente angular especifica o fator de correção, ou seja, o sub-registro de óbitos e o coeficiente de interseção determina a taxa de crescimento da população de estudo.

O Método de Preston e col..$^{10}$ também faz uso da distribuição de óbitos e da população por idade cuja informação primária se baseia nas relações da população estável e fornece como estimativa a cobertura dos registros de óbitos.

O Método de Courbage e Fargues ${ }^{2}$ pressupõe que existe uma idade além da qual a taxa de sub-registro de óbitos difere muito pouco de um grupo etário para outro; que a estrutura etária das taxas de mortalidade observadas é similar a um conjunto de taxas de mortalidade por idade específica padrão. Desta forma, o conhecimento da distribuição etária dos óbitos pode ser relacionado a um modelo padrão, possibilitando a estimativa do verdadeiro nível da mortalidade. $\mathrm{O}$ fator de correção do sub-registro de óbitos é obtido pela comparação entre a taxa de mortalidade de uma tábua de vida ${ }^{12,13}$ e a taxa de mortalidade calculada a partir dos dados registrados.

Com o propósito de assegurar maior precisão, confiança e consistência no resultado, selecionou-se um único fator de correção para cada estado, entre os resultados obtidos da aplicação dos três métodos. Para isso adotaram-se os seguintes critérios:

1 - Média aritmética dos fatores de correção cuja diferença entre si não ultrapassasse $10 \%$.

2 - Quando os fatores de correção ultrapassassem 10\%, levou-se em conta os seguintes aspectos: o diferencial por sexo (quanto menor mais apropriado) e o grupo etário no qual o fator de correção vigorou (quanto mais amplo, mais confiável a estimativa).

\section{RESU LTADO S E DISCUSSÃO}

\section{Dados Populacionais}

Os três índices foram aplicados para todos os estados brasileiros, cujos resultados da classificação para os da ONU e os de Whipple são encontrados na Tabela 1; os resultados da aplicação do Índice de Myers para o Brasil são apresentados na Tabela 2.As classificações adotadas para os três índices encontram-se no Anexo 1 e se referem a uma proposta de compatibilização entre eles fornecida pelas Nações Unidas ${ }^{8}$.

As comparações revelam claramente que o nível geral da qualidade dos dados por faixa etária para o Brasil e as unidades da federação foi alto para o ano de 1990, em ambos os sexos. Para 1980, no entanto, algumas irregularidades parecem ter ocorrido para a maioria dos estados do Norte e do Nordeste, destacando-se os estados do Rio Grande do Norte, 
Paraíba e Ceará, como de qualidade duvidosa. Para as demais Unidades da Federação (UF), os resultados das classificações parecem indicar populações livres de sérios erros nos registros das idades.
Desta forma, a distribuição por idade e sexo das populações fornecem um grau de confiabilidade aceitável das declarações de idade para a maioria dos estados, o qual não exige ajustamentos ou

Tabela 1 - Classificação dos Índices O NU e W hipple para os estados brasileiros, por sexo: 1980/1990.

\begin{tabular}{|c|c|c|c|c|c|c|}
\hline \multirow{3}{*}{$\begin{array}{l}\text { Unidades } \\
\text { da Federação }\end{array}$} & \multirow{2}{*}{\multicolumn{2}{|c|}{$\begin{array}{c}\text { Classificação } \\
\text { ONU }\end{array}$}} & \multicolumn{4}{|c|}{$\begin{array}{c}\text { Classificação } \\
\text { Whipple }\end{array}$} \\
\hline & & & \multicolumn{2}{|c|}{ Masc. } & \multicolumn{2}{|c|}{ Fem. } \\
\hline & 1980 & 1990 & 1980 & 1990 & 1980 & 1990 \\
\hline Roraima (RO) & imp. & Prc. & aprx. & pre. & grs. & prc. \\
\hline Acre $(A C)$ & imp. & Prc. & grs. & p.prc. & grs. & p.pre. \\
\hline Amazonas (AM) & imp. & Prc. & aprx. & p.prc. & aprx. & p.prc. \\
\hline Roraima (RR) & - & - & - & - & - & - \\
\hline Pará (PA) & imp. & prc. & aprx. & p.prc. & aprx. & p.prc. \\
\hline Amapá (AP) & - & - & - & - & - & - \\
\hline Tocantins (TO ) & - & - & - & - & - & - \\
\hline Maranhão (MA) & imp. & prc. & grs. & aprx. & grs. & aprx. \\
\hline Piauí (PI) & imp. & prc. & aprx. & prc. & aprx. & prc. \\
\hline Ceará (CE) & alt. imp. & prc. & aprx. & pre. & aprx. & prc. \\
\hline Rio Grande do Norte (RN) & alt. imp. & prc. & aprx. & pre. & aprx. & prc. \\
\hline Paraíba (PB) & alt. imp. & prc. & aprx. & pre. & aprx. & p.prc. \\
\hline Pernambuco (PE) & prc. & prc. & aprx. & prc. & aprx. & p.prc. \\
\hline Alagoas (AL) & imp. & prc. & grs. & aprx. & grs. & aprx. \\
\hline Sergipe (SE) & imp. & prc. & aprx. & p.pre & aprx. & p.prc. \\
\hline Bahia (BA) & imp. & prc. & aprx. & p.pre & aprx. & p.prc. \\
\hline M inas G erais (M G ) & prc. & prc. & aprx. & prc & aprx. & pre. \\
\hline Espírito Santo (ES) & prc. & prc. & p.prc. & p.pre & prc. & \\
\hline Rio de Janeiro (RJ) & prc. & prc. & p.prc. & prc & prc. & prc \\
\hline São Paulo (SP) & prc. & prc. & p.prc. & prc & p.pre & prc \\
\hline Paraná (PR) & imp. & prc. & p.prc. & prc & p.pre & prc \\
\hline Santa Catarina (SC) & prc. & prc. & p.prc. & prc & p.pre & prc \\
\hline Rio Grande do Sul (RS) & pre. & prc. & p.prc. & prc & prc & prc \\
\hline M ato Grosso do Sul (MS) & - & - & - & - & - & - \\
\hline M ato Grosso (MT) & imp. & prc. & aprx. & prc & aprx & prc \\
\hline Goiás (GO) & prc. & prc & aprx. & prc & aprx & prc \\
\hline Distrito Federal (DF) & - & - & - & - & - & - \\
\hline Brasil & prc. & prc. & aprx. & prc. & aprx. & prc. \\
\hline
\end{tabular}

prc. - precisos, p.prc. - pouco precisos, imp. - imprecisos, alt. imp. - altamente imprecisos, grs - grosseiros, aprx - aproximados

O bs: O Distrito Federal, os novos estados e os ex-territórios não foram considerados.

Tabela 2 - Índice de Myers e classificação do Índice para o Brasil : 1980/1990.

\begin{tabular}{lccccc}
\hline \multirow{2}{*}{ Sexo } & \multicolumn{2}{c}{ Índice } & & \multicolumn{2}{c}{ Classificação } \\
\cline { 2 - 3 } \cline { 5 - 6 } & 1980 & 1990 & & 1980 & 1990 \\
\hline Masculino & 4,19 & 1,38 & & baixa atração & baixa atração \\
Feminino & 4,50 & 1,47 & & baixa atração & baixa atração \\
\hline Total & 4,31 & 1,32 & & baixa atração & baixa atração \\
\hline
\end{tabular}

Tabela 3 - Ó bitos observados e esperados por fontes de dados segundo sexo para o Brasil: 1980/1990.

\begin{tabular}{|c|c|c|c|c|c|c|c|c|}
\hline \multirow{3}{*}{ Ó bitos } & \multicolumn{4}{|c|}{ M inistério da Saúde } & \multicolumn{4}{|c|}{ FIBGE } \\
\hline & \multicolumn{2}{|c|}{1980} & \multicolumn{2}{|c|}{1990} & \multicolumn{2}{|c|}{1980} & \multicolumn{2}{|c|}{1990} \\
\hline & $M$ & $\mathrm{~F}$ & $M$ & $\mathrm{~F}$ & $M$ & $\mathrm{~F}$ & $M$ & $\mathrm{~F}$ \\
\hline Fator de correção & 1,30 & 1,34 & 1,18 & 1,21 & 1,17 & 1,27 & 1,13 & 1,19 \\
\hline Ó bitos observados & 420.795 & 309.832 & 477.599 & 330.715 & 465.268 & 332.349 & 496.801 & 336.816 \\
\hline Ó bitos esperados & 547.033 & 415.175 & 563.567 & 400.165 & 544.364 & 422.083 & 561.385 & 400.811 \\
\hline
\end{tabular}


suavizações. Graduações na distribuição por idade somente eliminariam o efeito genuíno das mudanças demográficas ocorridas nas regiões consideradas com boa qualidade. Ademais, o agrupamento das idades em grupos qüinqüenais elimina a maioria das irregularidades devido à preferência digital. Este tipo de agrupamento é, deste modo, muito usado nos estudos etários que envolvem o conhecimento dos valores populacionais.

\section{Comparação dos Registros de Óbitos Provenientes de Fontes Distintas}

As estatísticas de óbitos provenientes da FIBGE e do MS são investigadas para o Brasil. Aplicados os três métodos de avaliação do sub-registro, adotados no presente trabalho, três estimativas foram obtidas, cujos critérios para seleção do fator de correção mais plausível são discutidos posteriormente. ATabela 3 mostra que os fatores de correção estimados em ambos os sexos, usando os dados do
MS, foram mais sub-registrados do que os da FIBGE, embora a fonte original de captação dos dados seja a mesma, os cartórios. Para 1980, a discrepância foi maior que em 1990 e, entre os sexos, o diferencial foi maior para os homens. As duas fontes revelam que houve um diferencial por sexo, sendo os homens menos sub-registrados do que as mulheres. A coerência dos resultados provenientes das duas fontes também pode ser constatada através da magnitude dos óbitos esperados para ambos os sexos. As diferenças absolutas não ultrapassaram 7.000 óbitos esperados, ou seja, $1,7 \%$, valor que recai numa margem de tolerância aceitável.

Os fatores de correção aplicados nas duas fontes de dados referem-se à idade de 5 anos e mais, conforme estabelecem os pressupostos das técnicas usadas. Sabendo-se que os sub-registros nas idades inferiores aos 5 anos são usualmente superiores aos das demais idades, aplica-se aqui, o mesmo fator de correção, uniformemente, para todas as idades, com
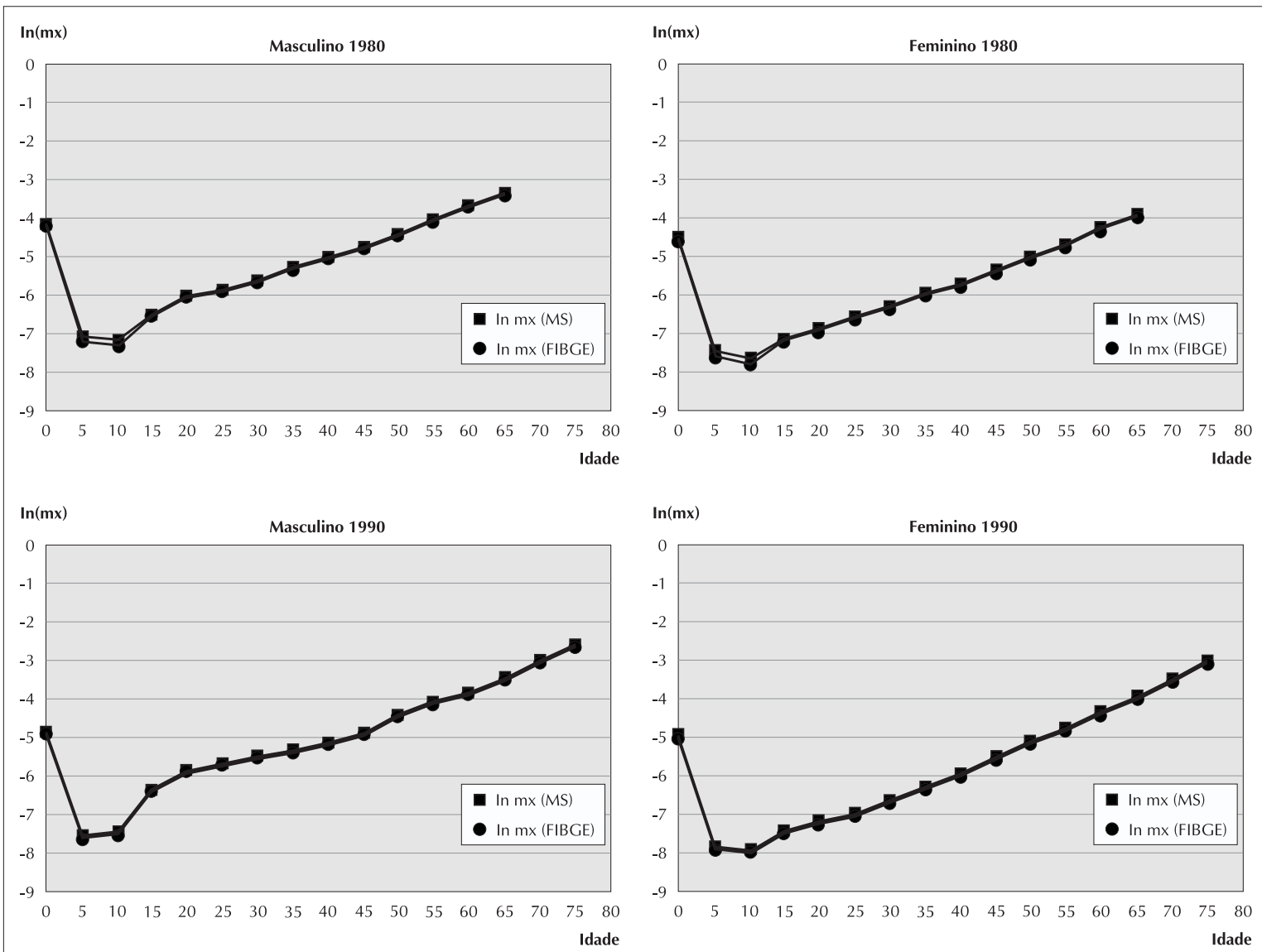

Figura - Log das taxas específicas de mortalidade $(\mathrm{mx})$ observada para o Brasil. 
Tabela 4 - Fatores de correção segundo diversas técnicas para os estados do Brasil por sexo e grupo etário: 1990

\begin{tabular}{|c|c|c|c|c|c|c|c|}
\hline \multirow{2}{*}{ UF } & & \multicolumn{3}{|c|}{ Fator de correção* } & \multicolumn{3}{|c|}{ Grupo etário* } \\
\hline & Sexo & Brass & Preston & $C \& F$ & Brass & Preston & $C \& F$ \\
\hline \multirow[t]{2}{*}{ RO } & $M$ & 1,54 & 1,18 & 1,30 & $45-65$ & $25-60$ & $10-60$ \\
\hline & $\mathrm{F}$ & 1,69 & 1,24 & 1,35 & $45-65$ & $35-65$ & $10-60$ \\
\hline \multirow[t]{2}{*}{$A C$} & M & 1,19 & 1,24 & 1,43 & $30-75$ & $35-80+$ & $10-50$ \\
\hline & $\mathrm{F}$ & 1,23 & 1,28 & 1,56 & $30-75$ & $10-60$ & $5-50$ \\
\hline \multirow[t]{2}{*}{ AM } & M & 1,41 & 1,27 & 1,40 & $55-70$ & $5-80$ & $5-50$ \\
\hline & $\mathrm{F}$ & 1,41 & 1,28 & 1,44 & $50-65$ & $35-70$ & $10-60$ \\
\hline \multirow[t]{2}{*}{ RR } & M & 1,54 & 1,52 & 1,96 & $30-70$ & $25-60$ & $1-60$ \\
\hline & $\mathrm{F}$ & 1,46 & 1,58 & 2,10 & $30-75$ & $25-60$ & $1-60$ \\
\hline \multirow[t]{2}{*}{ PA } & M & 1,60 & 1,15 & 1,29 & $25-40$ & $10-70$ & $5-60$ \\
\hline & $\mathrm{F}$ & $*$ & 1,12 & 1,39 & $*$ & $15-60$ & $5-50$ \\
\hline \multirow[t]{2}{*}{ AP } & M & $*$ & 1,09 & 1,40 & $*$ & $15-50$ & $5-50$ \\
\hline & $\mathrm{F}$ & $*$ & 1,15 & 1,44 & $*$ & $10-70$ & $10-60$ \\
\hline \multirow[t]{2}{*}{ MA } & $M$ & 1,55 & 1,78 & 2,09 & $30-55$ & $15-60$ & $1-50$ \\
\hline & $\mathrm{F}$ & 1,64 & 2,17 & 2,24 & $40-50$ & $5-65$ & $1-50$ \\
\hline \multirow[t]{2}{*}{ PI } & $M$ & 1,45 & 1,47 & 1,60 & $55-75$ & $20-70$ & $1-60$ \\
\hline & $\mathrm{F}$ & 1,68 & 1,75 & 1,50 & $30-75$ & $5-65$ & $1-60$ \\
\hline \multirow[t]{2}{*}{ CE } & $M$ & 1,28 & 1,25 & 1,32 & $30-70$ & $20-60$ & $1-60$ \\
\hline & $\mathrm{F}$ & 1,37 & 1,45 & 1,85 & $30-60$ & $15-60$ & $1-60$ \\
\hline \multirow[t]{2}{*}{ RN } & $M$ & 1,47 & 1,22 & 1,26 & $55-75$ & $25-60$ & $1-60$ \\
\hline & $\mathrm{F}$ & 1,49 & 1,42 & 1,49 & $40-75$ & $5-60$ & $1-60$ \\
\hline \multirow[t]{2}{*}{ PB } & $M$ & 1,20 & 1,28 & 1,26 & $30-75$ & $30-60$ & $1-50$ \\
\hline & $\mathrm{F}$ & 1,26 & 1,30 & 1,28 & $25-70$ & $15-70$ & $1-60$ \\
\hline \multirow[t]{2}{*}{ PE } & $M$ & 1,15 & 1,14 & 1,43 & $35-75$ & $30-60$ & $1-60$ \\
\hline & $\mathrm{F}$ & 1,14 & 1,18 & 1,21 & $30-75$ & $30-65$ & $1-50$ \\
\hline \multirow[t]{2}{*}{$A L$} & $M$ & 1,24 & 1,16 & 1,50 & $55-75$ & $30-60$ & $25-65$ \\
\hline & $\mathrm{F}$ & 1,27 & 1,18 & 1,21 & $50-65$ & $0-60$ & $5-50$ \\
\hline \multirow[t]{2}{*}{ SE } & $M$ & $*$ & 1,06 & 1,14 & $*$ & $35-60$ & $1-60$ \\
\hline & $\mathrm{F}$ & 1,10 & 1,12 & 1,19 & $50-70$ & $25-60$ & $5-60$ \\
\hline BA & $M$ & 1,15 & 1,19 & 1,31 & $30-75$ & $20-60$ & $1-60$ \\
\hline & $\mathrm{F}$ & 1,19 & 1,25 & 1,41 & $30-75$ & $5-60$ & $1-60$ \\
\hline MG & $M$ & 1,10 & 1,10 & 1,23 & $30-75$ & $10-70$ & $10-50$ \\
\hline & $\mathrm{F}$ & 1,16 & 1,15 & 1,25 & $30-75$ & $20-55$ & $5-50$ \\
\hline ES & $M$ & 1,08 & 1,06 & 1,13 & $45-70$ & $10-75$ & $5-60$ \\
\hline & $\mathrm{F}$ & 1,08 & 1,09 & 1,18 & $30-70$ & $25-70$ & $1-60$ \\
\hline RJ & $M$ & 1,08 & 1,06 & 1,07 & $30-75$ & $25-50$ & $5-60$ \\
\hline & $\mathrm{F}$ & 1,11 & 1,11 & 1,13 & $35-75$ & $30-55$ & $1-50$ \\
\hline SP & $M$ & 1,07 & 1,01 & 1,15 & $35-75$ & $30-70$ & $1-50$ \\
\hline & $\mathrm{F}$ & 1,05 & 1,06 & 1,13 & $60-75$ & $30-70$ & $1-50$ \\
\hline PR & $M$ & 1,11 & 1,09 & 1,12 & $35-75$ & $15-70$ & $1-60$ \\
\hline & $\mathrm{F}$ & 1,20 & 1,15 & 1,15 & $30-75$ & $25-70$ & $1-60$ \\
\hline SC & $M$ & 1,07 & 1,05 & 1,07 & $30-75$ & $35-75$ & $1-60$ \\
\hline & $\mathrm{F}$ & 1,02 & 1,18 & 1,17 & $40-60$ & $25-55$ & $1-60$ \\
\hline RS & $M$ & 1,11 & 1,10 & 1,10 & $30-75$ & $25-60$ & $5-60$ \\
\hline & $\mathrm{F}$ & 1,11 & 1,18 & 1,14 & $35-60$ & $25-55$ & $1-60$ \\
\hline MS & $M$ & 1,16 & 1,16 & 1,23 & $30-75$ & $25-65$ & $5-50$ \\
\hline & $\mathrm{F}$ & 1,22 & 1,37 & 1,30 & $30-70$ & $20-70$ & $10-50$ \\
\hline MT & $M$ & 1,23 & 1,25 & 1,36 & $40-75$ & $30-70$ & $1-60$ \\
\hline & $\mathrm{F}$ & 1,22 & 1,32 & 1,40 & $30-75$ & $30-65$ & $1-60$ \\
\hline GO & $M$ & 1,22 & 1,19 & 1,20 & $35-60$ & $10-70$ & $1-60$ \\
\hline & $\mathrm{F}$ & 1,15 & 1,26 & 1,21 & $30-55$ & $20-60$ & $10-50$ \\
\hline$D F$ & $M$ & 1,04 & 1,04 & 0,92 & $30-70$ & $25-55$ & $15-60$ \\
\hline & $\mathrm{F}$ & 1,04 & 1,05 & 1,12 & $30-70$ & $30-65$ & $15-60$ \\
\hline Brasil & $M$ & 1,12 & 1,14 & 1,28 & $40-75$ & $1-50$ & $1-60$ \\
\hline & $\mathrm{F}$ & 1,18 & 1,20 & 1,25 & $30-75$ & $25-55$ & $1-50$ \\
\hline
\end{tabular}


o objetivo de calcular as taxas de mortalidade corrigidas. Portanto, ao assumir que a magnitude dos erros na estimação do sub-registro para os menores de 5 anos é o mesmo para ambas as fontes de dados, comete-se um erro na estimação da taxa de mortalidade para as primeiras idades. Sobre o padrão etário da mortalidade brasileira, estes erros estariam presentes nas duas fontes de dados. Assumindo que eles possuem a mesma magnitude não se inviabiliza a comparação para efeito de entendimento do comportamento etário da mortalidade.

As taxas de mortalidade específicas por grupos etários foram calculadas e ajustadas pelos fatores de correção correspondentes a cada sexo, utilizando as duas fontes para fins comparativos. A Figura mostra o seu comportamento para o Brasil, por sexo, respectivamente para 1980 e 1990 quando são tomados os logaritmos das probabilidades de morte.

Observa-se que o padrão etário nas duas fontes praticamente não apresentou diferença para $1980 \mathrm{e}$ para 1990. Esta similaridade sugere que o padrão da mortalidade no Brasil não revela diferenças nas duas fontes de dados investigadas; mais ainda, o comportamento das curvas não aponta irregularidades que sugiram modificações ou suavizamentos. Portanto, as taxas de mortalidade, uma vez corrigidas, possibilitam construir tábuas de vida, fornecendo razoáveis estimativas de indicadores importantes como a esperança de vida ao nascer e a taxa de mortalidade infantil por qualquer uma das fontes consideradas.

\section{Cobertura dos Registros de Óbitos}

Uma vez que ambas as fontes de dados produziram padrões de mortalidade semelhantes quando corrigidos pelos respectivos fatores de correção, não há necessidade de aplicar os métodos em ambas as fontes para os estados brasileiros. A opção recaiu na escolha dos dados de registros de óbitos produzidos pela FIBGE para a aplicação dos três métodos. As razões são várias: eles cobrem um período muito maior, são de mais fácil acesso, representam as estatísticas oficiais nas comparações internacionais e apresentaram uma cobertura nacional maior do que a dos dados produzidos pelo MS. Assim, utilizando os dados da FIBGE produziramse os resultados mostrados na Tabela 4, que também mostra os grupos etários onde vigoram os fatores de correção encontrados.
Tabela 5 - Fatores de correção e cobertura dos registros de óbitos selecionados para os estados do Brasil por sexo: 1990.

\begin{tabular}{|c|c|c|c|c|}
\hline UF & Sexo & Freq. & $\begin{array}{c}\text { Cobertura } \\
(\%)\end{array}$ & $\begin{array}{c}\text { Média das } \\
\text { técnicas }\end{array}$ \\
\hline \multirow[t]{2}{*}{ RO } & $M$ & 1,54 & 64,94 & $B$ \\
\hline & $\mathrm{F}$ & 1,64 & 60,98 & B \\
\hline \multirow[t]{2}{*}{$A C$} & $M$ & $>1,40$ & $<71,43$ & $C \& F$ \\
\hline & $\mathrm{F}$ & $>1,50$ & $<66,67$ & $C \& F$ \\
\hline \multirow[t]{2}{*}{$A M$} & $M$ & 1,40 & 71,43 & $B / C \& F$ \\
\hline & $\mathrm{F}$ & 1,43 & 69,93 & $\mathrm{~B} / \mathrm{C} \& \mathrm{~F}$ \\
\hline \multirow[t]{2}{*}{$\mathrm{RR}$} & M & 1,53 & 65,36 & B / P \\
\hline & $\mathrm{F}$ & 1,52 & 65,79 & $B / P$ \\
\hline \multirow[t]{2}{*}{ PA } & $M$ & 1,59 & 62,89 & B \\
\hline & $\mathrm{F}$ & $>1,60$ & $<62,5$ & $*$ \\
\hline \multirow[t]{2}{*}{$A P$} & $M$ & 1,40 & 71,43 & $C \& F$ \\
\hline & $\mathrm{F}$ & 1,44 & 69,44 & $C \& F$ \\
\hline \multirow[t]{2}{*}{ MA } & $M$ & $>1,55$ & $<64,52$ & B \\
\hline & $\mathrm{F}$ & $>1,64$ & $<60,68$ & B \\
\hline \multirow[t]{2}{*}{$\mathrm{PI}$} & $M$ & 1,46 & 68,49 & $B / P$ \\
\hline & $\mathrm{F}$ & $>1,50$ & $<66,67$ & $C \& F$ \\
\hline \multirow[t]{2}{*}{$C E$} & M & 1,28 & 78,12 & B \\
\hline & $\mathrm{F}$ & 1,37 & 72,99 & B \\
\hline \multirow[t]{2}{*}{ RN } & M & 1,36 & 73,53 & $B / P / C \& F$ \\
\hline & $\mathrm{F}$ & 1,45 & 68,97 & $B / P / C \& F$ \\
\hline \multirow[t]{2}{*}{ PB } & M & 1,24 & 80,64 & $B / P / C \& F$ \\
\hline & $\mathrm{F}$ & 1,28 & 78,12 & $B / P / C \& F$ \\
\hline \multirow[t]{2}{*}{$P E$} & $M$ & 1,14 & 87,72 & B / P \\
\hline & $\mathrm{F}$ & 1,12 & 89,29 & $B / P$ \\
\hline \multirow[t]{2}{*}{$A L$} & $M$ & 1,20 & 83,33 & $B / P$ \\
\hline & $\mathrm{F}$ & 1,23 & 81,30 & B / P \\
\hline \multirow[t]{2}{*}{ SE } & $M$ & 1,10 & 90,91 & $\mathrm{P} / \mathrm{C} \& \mathrm{~F}$ \\
\hline & $\mathrm{F}$ & 1,15 & 86,96 & $\mathrm{P} / \mathrm{C} \& \mathrm{~F}$ \\
\hline \multirow[t]{2}{*}{ BA } & $M$ & 1,15 & 86,96 & B \\
\hline & $\mathrm{F}$ & 1,22 & 81,97 & $B / P$ \\
\hline \multirow[t]{2}{*}{ MG } & M & 1,10 & 90,91 & $B / P$ \\
\hline & $\mathrm{F}$ & 1,15 & 86,96 & $B / P$ \\
\hline \multirow[t]{2}{*}{ ES } & $M$ & 1,07 & 93,46 & $B / P$ \\
\hline & $\mathrm{F}$ & 1,08 & 92,59 & B / P \\
\hline \multirow[t]{2}{*}{ RJ } & $M$ & 1,07 & 93,46 & $\mathrm{~B} / \mathrm{P} / \mathrm{C} \& \mathrm{~F}$ \\
\hline & $\mathrm{F}$ & 1,11 & 90,09 & $B / P / C \& F$ \\
\hline \multirow[t]{2}{*}{ SP } & $M$ & 1,04 & 96,15 & B / P \\
\hline & $\mathrm{F}$ & 1,05 & 95,24 & B / P \\
\hline PR & $M$ & 1,10 & 90,91 & $\mathrm{~B} / \mathrm{P} / \mathrm{C} \& \mathrm{~F}$ \\
\hline & $\mathrm{F}$ & 1,17 & 85,47 & $B / P / C \& F$ \\
\hline SC & $M$ & 1,06 & 94,34 & $\mathrm{~B} / \mathrm{P} / \mathrm{C} \& \mathrm{~F}$ \\
\hline & $\mathrm{F}$ & 1,08 & 92,59 & $B / P / C \& F$ \\
\hline RS & $M$ & 1,10 & 90,91 & $B / P / C \& F$ \\
\hline & $\mathrm{F}$ & 1,12 & 89,29 & $B / C \& F$ \\
\hline MS & $M$ & 1,20 & 83,33 & B / P \\
\hline & $\mathrm{F}$ & 1,26 & 79,37 & $B / P$ \\
\hline MT & $M$ & 1,24 & 80,64 & $B / P$ \\
\hline & $\mathrm{F}$ & 1,27 & 78,74 & B / P \\
\hline GO & M & 1,20 & 83,33 & $B / P / C \& F$ \\
\hline & $\mathrm{F}$ & 1,18 & 84,75 & $\mathrm{~B} / \mathrm{C} \& \mathrm{~F}$ \\
\hline DF & $M$ & 1,04 & 96,15 & B / P \\
\hline & $\mathrm{F}$ & 1,04 & 96,15 & $B / P$ \\
\hline Brasil & $M$ & 1,13 & 88,50 & $B / P$ \\
\hline & $\mathrm{F}$ & 1,19 & 84,03 & $B / P$ \\
\hline
\end{tabular}

Nota : $f=$ freqüência; $B$ - Brass' ${ }^{1}$ P - Presto e col. ${ }^{10} ; C \& F$ - Courbage $\&$ Fargues $^{2}$ 
No Anexo 2 encontram-se os modelos de tábuas de vida empregadas como referência, por serem os que melhor se ajustaram aos dados observados para todas as UF, por sexo, quando o método de Courbage e Fargues ${ }^{2}$ foi aplicado.

\section{Escolha do Fator de Correção}

A Tabela 5 mostra, por sexo, o fator de correção selecionado, a cobertura de óbitos em percentagem e os métodos utilizados na definição do fator de correção selecionado.

Para o Brasil, em 1990, os resultados gerados pelos métodos de Brass $^{1}$ e Preston e Coale ${ }^{10}$ foram muito próximos para ambos os sexos (Tabela 4), de forma que a média aritmética foi extraída, ficando os fatores de correção em torno de 1,13 para o sexo masculino e de 1,19 para o femininino, os quais correspondem a $88,5 \%$ e $84,03 \%$, respectivamente, de cobertura dos registros de óbitos.

De modo geral, para os estados, os métodos de Brass $^{1}$ e Preston et al.$^{10}$ produziram os resultados mais próximos. Em 11 estados houve uma diferença menor que $5 \%$ entre pelo menos dois métodos, para ambos os sexos e, em 4 estados, para pelo menos um dos sexos o que mostra uma boa concordância entre os dois métodos. Em 9 outros estados, essa diferença foi entre $5 \%$ e $10 \%$.

A Tabela 6 mostra classificação da qualidade da cobertura dos registros de óbitos, adotado pelo CELADE6 . De acordo com esta classificação, apenas o Distrito Federal, o Estado de Sergipe e todos os estados da região Sul e Sudeste do País revelaram uma cobertura "boa" em 1990 para o sexo masculino, ou seja, superior a $90 \%$. Na direção contrária situaram-se, nas

Tabela 6 - Classificação dos estados brasileiros segundo o grau de cobertura dos registros de óbitos por sexo: 1990.

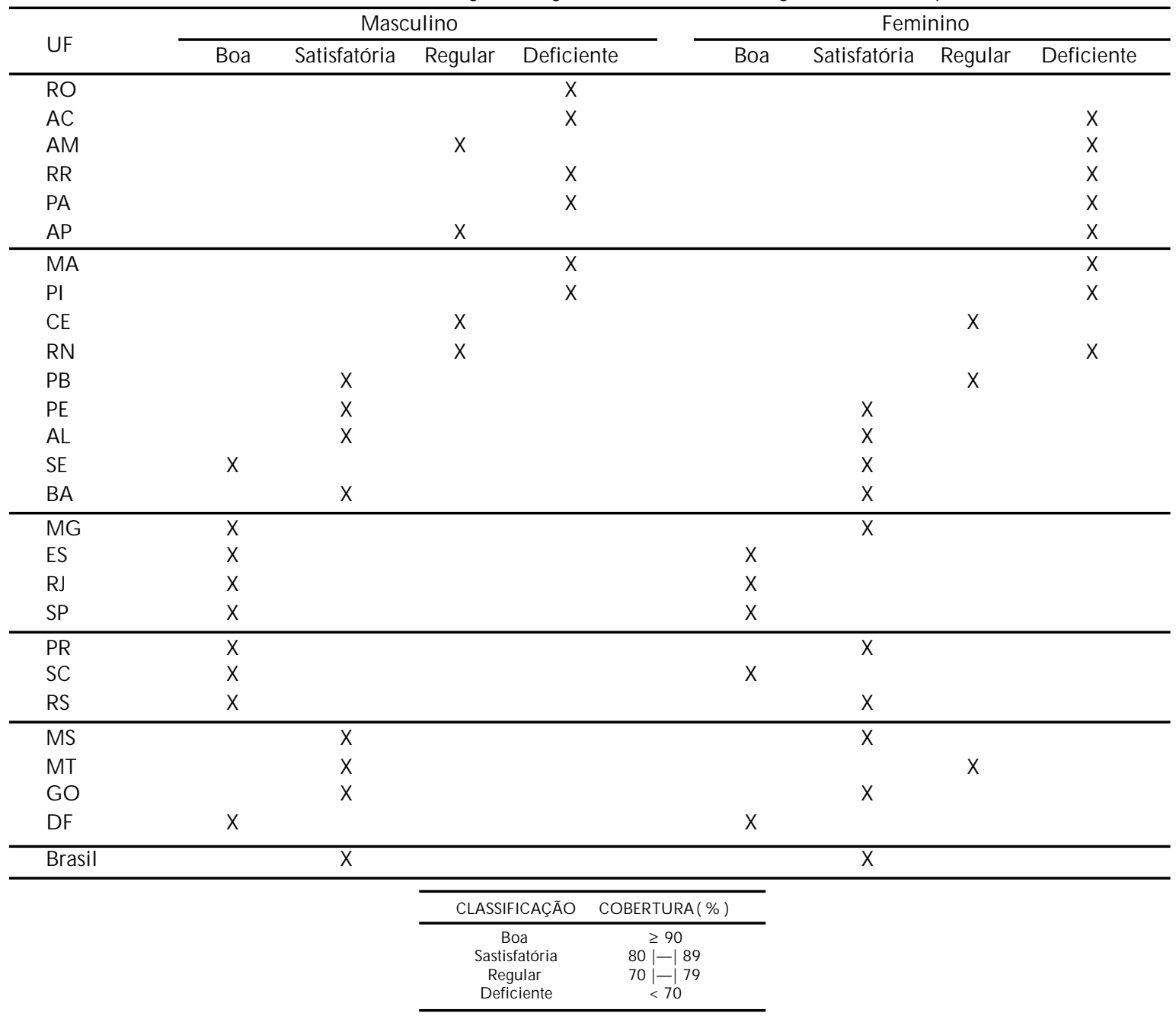


regiões Norte, os estados do Acre, Pará, Roraima e Rondônia e no Nordeste, os estados do Maranhão e do Piauí.

O quadro para o sexo feminino, em 1990, para esta categoria, não se mostrou muito diferente daquele para o sexo masculino, no entanto, foi menor o número de estados com qualidade de cobertura "boa". Apenas os estados do Rio de Janeiro, Espírito Santo, São Paulo, Santa Catarina e o Distrito Federal encontravam-se nesta situação. E na categoria com cobertura de registro de óbitos de qualidade "deficiente" encontravam se todos os estados do Norte e, no Nordeste, os estados do Maranhão, Piauí e Rio Grande do Norte. Todos os demais estados, em ambos os sexos, encontravamse, portanto, em uma situação de "satisfatória" ou "regular".

Na categoria "satisfatória", com cobertura dos registros de óbitos entre 80 e $89 \%$ figuraram no Nordeste, os estados de Pernambuco, Paraíba, Alagoas, Bahia e Sergipe para ambos os sexos com exceção da Paraíba (feminino) como "regular" e de Sergipe (masculino) como "boa". Ou seja, no Nordeste, a maioria dos estados já se encontrava em uma situação no mínimo satisfatória, resultando em um quadro que sugere que as estatísticas para a maioria dos estados brasileiros captaram, pelo menos regularmente, os padrões de mortalidade e, conseqüentemente, ocuparam o seu papel de fornecer referências úteis para estimar níveis de mortalidade satisfatórios.

Ressalta-se o diferencial por sexo, em favor do sexo masculino, com mais altos registros para quase todas as UF. Excetuam-se os estados de Goiás e Pernambuco onde a ordem foi inversa, mas com diferencial de pequena magnitude, e o Estado de Roraima e Distrito Federal, com similares níveis de cobertura.

Achados nesta direção foram encontrados por Paes $^{9}$ em uma investigação similar ao comparar as coberturas dos registros de óbitos para 1959 e para 1980 para vários estados e o País como um todo. Para o Brasil, no ano de 1980, Paes ${ }^{9}$ estimou uma cobertura em torno de $85 \%$ para o sexo masculino e de $79 \%$ para o sexo feminino e, em 1959 , as coberturas foram de $63 \%$ e de $61 \%$ para o sexo masculino e feminino, respectivamente. Assim, é possível enunciar que no Brasil houve melhoras na cobertura dos registros de óbitos, havendo um diferencial importante entre os sexos que favoreceu uma maior cobertura para os homens.

\section{CONCLUSÕES}

Houve uma melhora significativa na qualidade dos dados censitários quanto à preferência digital de 1991 com relação a 1980, o que conduz a uma confiança maior nos indicadores que dependem da distribuição etária da população de 1991. Comparações feitas do padrão etário da mortalidade no Brasil entre os dados fornecidos pela FIBGE e pelo MS, não evidenciaram diferença no comportamento do padrão, mas sim nos níveis, os quais praticamente não se diferenciaram em 1990. Isto é, conhecendo-se o sub-registro dos óbitos divulgados pela FIBGE, admite-se que o sub-registro de óbitos do MS seja próximo. Portanto, os estudos que quantificam os óbitos por faixa etária encontram, pelo menos para o Brasil, respaldo nos dados da FIBGE, particularmente para 1990. E, esta informação pode ser útil para dimensionar o volume dos óbitos por faixa etária nos estudos que envolvem as causas de óbitos, já que apenas o MS fornece esses dados desagregados por causas específicas, idade e sexo.

A aplicação dos métodos de estimação do subregistro de óbitos mostrou que no Brasil e UF houve aumento considerável da cobertura dos óbitos no tempo, revelando diferenciais por sexo em favor do masculino e que a cobertura foi mais elevada para as UF das regiões Sul e Sudeste do País.

Constata-se, assim, uma melhoria na qualidade do sistema dos registros civis o que permite avaliar com mais segurança os indicadores deles derivados e, conseqüentemente, planejar melhor as ações voltadas para a saúde pública. Esta evidência sugere que não há motivos para negligenciar as estatísticas de óbitos no Brasil. A desculpa de que eles são de má qualidade não serve como pretexto para ignorálos. Para muitas regiões do Brasil, eles possuem poder explicativo suficiente para construir indicadores de mortalidade com satisfatória confiança. 


\section{REFERÊN CIAS}

1. Brass W. Methods for estimating fer tility and mortality from limited and defective data. Chapel Hill: International Program of Laboratories for Population Statistics; 1975. (Occasional Publication).

2. Courbage Y, Fargues P. A method for deriving mortality estimates from incomplete vital statistics. Popul Stud. 1979; $33: 165-80$

3. Fundação IBGE. Censo demográfico, 1980. Rio de Janeiro, 1983 ( $9^{\circ}$ Recenseamento Geral do Brasil).

4. Fundação IBGE. Censo demográfico 1991. Rio de Janeiro, 1994 (10 Recenseamento Geral do Brasil).

5. Fundação IBGE. Estatísticas do registro civil, 1979 a 1981, 1984 a 1986, 1989 a 1991. Rio de Janeiro, 1979/1991 (Publicação anual).

6. Jasper-Faijer D, Orellana H. Evaluacion del uso de las estadisticas vitales para estudios de causas de muerte en America Latina. Notas Población 1994; 22 (60):47-77.

7. Ministério da Saúde. Sistema de informação sobre mortalidade: 1979 - 1993. [CD-ROM]. Brasília: CENEPI, 1995.

8. Naciones Unidas. Métodos para evaluar la calidad de los datos básicos destinados a los calculos de la población. In: Manuales sobre metodos de calculo de la población. Nueva York; 1955 (Manual II, N²3, série A).

9. Paes NA. Model life representation for Brazilian mortality. London; 1993. [Tese de doutorado - London School of Hygiene and Tropical Medicine, University of London].

10. Preston S, Coale AJ, Trussell J, Wenstein M. Estimating the completeness of reporting of adult deaths in populations that are aproximately stable. Popul Index 1980; 46: 179-202.

11. Shryock H S, Siegel JS et al. The methods and materials of demography. In: Stockwell EG. Studies in population. Condensed Edition. San Diego (CA):

Academic Press, 1975.

12. United Nations. Model life tables for developing countries. New York; 1982. (Population Studies, 77).

13. United Nations. Manual X: indirect techniques for demographic estimation. New York; 1983. (Population Studies, 81).

\section{Anexo 1}

\section{CLASSIFICAÇÃO DOS ÍNDICES}

\begin{tabular}{lr}
\hline \multicolumn{2}{c}{ Nações Unidas } \\
\hline Categoria & Campo de valores do Índice \\
qualitativa & das N ações U nidas \\
\hline 1 - Dados precisos & INU $\leq 20$ \\
2 - Dados imprecisos & $20<$ INU $\leq 40$ \\
3 - Dados altamente imprecisos & INU $>40$ \\
\hline Fonte: Nações Unidas $^{8}$ &
\end{tabular}

\begin{tabular}{lc}
\hline \multicolumn{2}{c}{ Whipple } \\
\hline Categoria & Campo de valores do Índice \\
qualitativa & Whipple \\
\hline 1 - Dados precisos & $99<\mathrm{W}<105$ \\
2 - Dados pouco precisos & $104<\mathrm{W}<110$ \\
3 - Dados aproximados & $109<\mathrm{W}<125$ \\
4 - Dados grosseiros & $124<\mathrm{W}<175$ \\
5 - Dados muito grosseiros & $174<\mathrm{W}<501$ \\
\hline
\end{tabular}

Fonte: $\mathrm{N}$ ações Unidas ${ }^{8}$

\begin{tabular}{lc}
\hline \multicolumn{2}{c}{ Myers } \\
\hline $\begin{array}{l}\text { Categoria } \\
\text { qualitativa }\end{array}$ & Campo de valores do Índice \\
Myers
\end{tabular}




\section{Anexo 2}

MO DELOS DE TÁBU AS DE VIDA EMPREG ADOS NA

APLICAÇÃO DO MÉTODO DE COURBAGE E FARGUES

PARA O S ESTAD O S BRASILEIRO S PO R SEXO : 1990.

\begin{tabular}{|c|c|c|}
\hline Estado & Sexo & Modelo \\
\hline \multirow[t]{2}{*}{ RO } & $M$ & Latino \\
\hline & $\mathrm{F}$ & Latino \\
\hline \multirow[t]{2}{*}{$A C$} & $M$ & Latino \\
\hline & $\mathrm{F}$ & Latino \\
\hline \multirow[t]{2}{*}{$A M$} & $M$ & Latino \\
\hline & $\mathrm{F}$ & Latino \\
\hline \multirow[t]{2}{*}{$\mathrm{RR}$} & $M$ & Latino \\
\hline & $\mathrm{F}$ & Latino \\
\hline \multirow[t]{2}{*}{ PA } & $M$ & Latino \\
\hline & $\mathrm{F}$ & Latino \\
\hline \multirow[t]{2}{*}{$A P$} & $M$ & Latino \\
\hline & $\mathrm{F}$ & Latino \\
\hline \multirow[t]{2}{*}{ MA } & $M$ & Chileno \\
\hline & $\mathrm{F}$ & Latino \\
\hline \multirow[t]{2}{*}{$\mathrm{PI}$} & $M$ & Chileno \\
\hline & $\mathrm{F}$ & Latino \\
\hline \multirow[t]{2}{*}{$C E$} & $M$ & Latino \\
\hline & $\mathrm{F}$ & Latino \\
\hline \multirow[t]{2}{*}{ RN } & $M$ & Latino \\
\hline & $\mathrm{F}$ & Latino \\
\hline \multirow[t]{2}{*}{ PB } & $M$ & Latino \\
\hline & $\mathrm{F}$ & Latino \\
\hline \multirow[t]{2}{*}{ PE } & $M$ & Chileno \\
\hline & $\mathrm{F}$ & Chileno \\
\hline \multirow[t]{2}{*}{$A L$} & $M$ & Chileno \\
\hline & $\mathrm{F}$ & Latino \\
\hline \multirow[t]{2}{*}{ SE } & $M$ & Latino \\
\hline & $\mathrm{F}$ & Latino \\
\hline \multirow[t]{2}{*}{ BA } & $M$ & Latino \\
\hline & $\mathrm{F}$ & Latino \\
\hline \multirow[t]{2}{*}{$M G$} & $M$ & Chileno \\
\hline & $\mathrm{F}$ & Chileno \\
\hline \multirow[t]{2}{*}{ ES } & $M$ & Latino \\
\hline & $\mathrm{F}$ & Latino \\
\hline \multirow[t]{2}{*}{ RJ } & $M$ & Latino \\
\hline & $\mathrm{F}$ & Chileno \\
\hline \multirow[t]{2}{*}{$\mathrm{SP}$} & $M$ & Chileno \\
\hline & $\mathrm{F}$ & Chileno \\
\hline \multirow[t]{2}{*}{ PR } & $M$ & Chileno \\
\hline & $\mathrm{F}$ & Chileno \\
\hline \multirow[t]{2}{*}{$\mathrm{SC}$} & $M$ & Chileno \\
\hline & $\mathrm{F}$ & Chileno \\
\hline $\mathrm{RS}$ & $M$ & Chileno \\
\hline & $\mathrm{F}$ & Latino \\
\hline MS & $M$ & Latino \\
\hline & $\mathrm{F}$ & Latino \\
\hline $\mathrm{MT}$ & $M$ & Latino \\
\hline & $\mathrm{F}$ & Latino \\
\hline GO & $M$ & Latino \\
\hline & $\mathrm{F}$ & Latino \\
\hline DF & $M$ & Latino \\
\hline & $\mathrm{F}$ & Chileno \\
\hline Brasil & $\begin{array}{c}M \\
F\end{array}$ & $\begin{array}{l}\text { Chileno } \\
\text { Chileno }\end{array}$ \\
\hline
\end{tabular}

Nota: As Tábuas de Vida relativas aos modelos Latino e Chileno são encontradas em U nited $\mathrm{N}$ ations ${ }^{12}$ (1982). 